\title{
Interatomic Pair Interaction in Liquid Transition Metals
}

\author{
N.E. Dubinin \\ Institute of Metallurgy of the Ural Branch of the Russian Academy of Sciences \\ Amundsen st. 101, 620016 Ekaterinburg, Russia
}

\begin{abstract}
The Wills-Harrison pair interaction is investigated for liquid Fe on the base of the introduced relation between parameters of the model pseudopotential and $d$-electron characteristics. It is shown that the procedure suggested leads to the significant shift of the first minimum of the pair potential and increases the softness of its repulsive part.
\end{abstract}

PACS numbers: 61.25.Mv, 71.15.Dx, 71.20.Be

\section{Introduction}

Wills and Harrison (WH) [1] presented the effective pair potential for transition metals as a sum of two terms. (The first one arises due to $d$ electrons, while the second corresponds to the standard pair potential in the nearly-free-electron (NFE) theory and may be calculated on the base of the ion-s-electron interaction):

$$
\varphi_{\mathrm{WH}}(r)=\varphi_{d}(r)+\varphi_{\mathrm{NFE}}(r) \text {. }
$$

To calculate the $\varphi_{\mathrm{NFE}}(r)$ in liquid transition metals, it was suggested [2-4] to use the Bretonnet-Silbert (BS) local model pseudopotential (MP) [5] (in the last years, this MP has been successfully used for the liquid $\mathrm{Ag}-\mathrm{In}$ study $[6-8])$.

Up to now, the contributions $\varphi_{d}(r)$ and $\varphi_{\mathrm{NFE}}(r)$ were treated as independent of each other. Here, we suggest a consistency for them.

In Sect. 2, we furnish the Bretonnet-Silbert pseudopotential formalism. In Sect. 3, the $d$-electron part of the WH effective pair potential is described. In Sect. 4 , we introduce the relationship between proofs of Sects. 2 and 3. The influence of the relationship presented on the pair interaction in liquid iron is discussed in Sect. 5 .

\section{The Bretonnet-Silbert model potential}

The BS MP was constructed on the base of the Oli [9] approach: inside the sphere of radius $R_{\mathrm{c}}$, the MP is described by the two first terms of the Dirichlet series, while outside of the sphere, it has the Coulomb form (for the unscreened ion):

$$
\omega_{\mathrm{O}}(r)= \begin{cases}\sum_{n=1}^{2} B_{n} \exp \left(-\frac{r}{n R_{\mathrm{c}}}\right), & r \leq R_{\mathrm{c}} \\ -z e^{2} / r, & r \geq R_{\mathrm{c}}\end{cases}
$$

where $B_{1}, B_{2}$ - coefficients of the series $z$ - number of $s$ electrons per ion $e$ - the electron charge.

In the BS MP, the ion radius $R_{\mathrm{c}}$ in the Dirichlet series is changed on some phenomenological parameter $a$, and the coefficients $B_{1}, B_{2}$ should be chosen as to provide the continuity of the MP and its first derivative at $r=R_{\mathrm{c}}$ :

$$
\begin{aligned}
& \omega_{\mathrm{BS}}(r)= \\
& \qquad \begin{array}{ll}
B_{1} \exp (-r / a)+B_{2} \exp (-0.5 r / a), & r \leq R_{\mathrm{c}} \\
-z e^{2} / r, & r \geq R_{\mathrm{c}}
\end{array} \\
& B_{1}=\left(z e^{2} / R_{\mathrm{c}}\right)\left(1-2 a / R_{\mathrm{c}}\right) \exp \left(R_{\mathrm{c}} / a\right), \\
& B_{2}=\left(2 z e^{2} / R_{\mathrm{c}}\right)\left(a / R_{\mathrm{c}}-1\right) \exp \left(0.5 R_{\mathrm{c}} / a\right)
\end{aligned}
$$

According to the WH approach [1], $z$ in (2) and (3) is treated as an effective valence which is lower than the number of $s$ electrons in a single atom due to the $s-d$ hybridization.

\section{The Wills-Harrison $d$-electron contribution to the pair interaction}

To describe the density of $d$ states, $n_{d}(\varepsilon)$, the Friedel model [10] was used by WH:

$$
n_{d}(\varepsilon)=\left\{\begin{array}{lr}
10 / W_{d}, & \varepsilon_{d}-W_{d} / 2 \leq \varepsilon \leq \varepsilon_{d}+W_{d} / 2, \\
0, & \text { otherwise, }
\end{array}\right.
$$

where $W_{d}, \varepsilon_{d}$ - the width and the heavy center of the $d$ band, respectively.

The $d$-electron contribution to the internal energy per atom, $E_{d}$, may be calculated by standard way (in atomic units):

$$
\begin{aligned}
& E_{d}=\int_{\varepsilon_{d}-W_{d} / 2}^{\varepsilon_{\mathrm{F}}} n_{d}(\varepsilon) f(\varepsilon) \varepsilon \mathrm{d} \varepsilon= \\
& -\frac{1}{2} z_{d}\left(\frac{10-z_{d}}{10}\right) W_{d}+z_{d} \varepsilon_{d}=E_{b}+E_{\mathrm{c}},
\end{aligned}
$$

where $\varepsilon_{\mathrm{F}}$ - the Fermi energy, $z_{d}$ - the number of valence $d$ electrons per ion $\left(z_{d}=Z-z\right.$, where $Z$ is the total number of the valence $s$ and $d$ electrons), $f(\varepsilon)-$ the Fermi distribution function, $E_{b}$ and $E_{\mathrm{c}}$ are the contributions due to the $d$-band width and to the shift of its heavy center with respect to the $d$ level of single atom, respectively. These contributions were obtained in [11] by using the muffin-tin (MT) potential and the atomic 
sphere approximation:

$$
\begin{aligned}
E_{b} & =-\sqrt{3} z_{d}\left(\frac{10-z_{d}}{10}\right) \\
& \times \sqrt{4 \pi \rho \int_{0}^{\infty} V_{d}^{2}(r) g(r) r^{2} \mathrm{~d} r}, \\
E_{\mathrm{c}} & =z_{d} 2 \pi \rho \int_{0}^{\infty} \frac{225 r_{d}^{6}}{\pi^{2} r^{8}} g(r) r^{2} \mathrm{~d} r,
\end{aligned}
$$

where $\rho$ is the mean atomic density, $g(r)$ - the pair correlation function, $r_{d}$ - the $d$-state radius, which is the characteristic of a single atom, $V_{d}(r)$ - the effective potential of the $d-d$ interaction:

$$
V_{d}(r)=\frac{28.06 r_{d}^{3}}{\pi r^{5}}
$$

From (7), one gets the $\varphi_{\mathrm{c}}(r)$ contribution into pair interaction:

$$
\varphi_{\mathrm{c}}(r)=z_{d} \frac{225 r_{d}^{6}}{\pi^{2} r^{8}}
$$

From (6), it is impossible to derive the contribution into the pair interaction directly. An approximated expression was found by $\mathrm{WH}$ as a variational derivative $\delta\left(N E_{b}\right) / \delta V_{d}\left(\boldsymbol{r}_{m l}\right)$ (where $N$ is the whole number of atoms). If one accounts $d-d$ interactions only for the first coordination shell with coordination number $\nu$, then the contribution is

$$
\varphi_{b}(r)=-z_{d}\left(\frac{10-z_{d}}{10}\right)\left(\frac{12}{\nu}\right)^{1 / 2} V_{d}(r) .
$$

Thus, the total $d$-electron contribution into the pair interaction is

$$
\varphi_{d}(r)=\varphi_{b}(r)+\varphi_{\mathrm{c}}(r) .
$$

\section{Relations between MP parameters and $d$-electron characteristics}

In general form, the phase shift, $\eta_{l}(\varepsilon)$, for the electron with energy $\varepsilon$ may be written for an arbitrary spherical potential, $\omega(r)$, as [12]:

$$
\tan \eta_{l}(\varepsilon)=-\sqrt{\varepsilon} \int_{0}^{\infty} j_{l}(r \sqrt{\varepsilon}) R_{l}(r) \omega(r) r^{2} \mathrm{~d} r,
$$

where $j_{l}(x)$ is the Bessel function, $l$ - the orbital quantum number, $R_{l}(r)$ - the radial part of the solution of the Schrödinger equation for the potential considered:

$$
R_{l}(r)=j_{l}(r \sqrt{\varepsilon})-\tan \eta_{l}(\varepsilon) n_{l}(r \sqrt{\varepsilon}),
$$
where $n_{l}(x)$ is the Neumann function.

From (12), (13) one gets

$$
\begin{aligned}
& \tan \eta_{l}(\varepsilon)=\frac{X}{1-Y}, \\
& X=-\sqrt{\varepsilon} \int_{0}^{\infty} j_{l}^{2}(r \sqrt{\varepsilon}) \omega(r) r^{2} \mathrm{~d} r, \\
& Y=\sqrt{\varepsilon} \int_{0}^{\infty} j_{l}(r \sqrt{\varepsilon}) n_{l}(r \sqrt{\varepsilon}) \omega(r) r^{2} \mathrm{~d} r .
\end{aligned}
$$

For $l=2$ ( $d$-electron state):

$$
j_{2}(x)=\left(\frac{3}{x^{3}}-\frac{1}{x}\right) \sin (x)-\frac{3}{x^{2}} \cos (x),
$$

$$
n_{2}(x)=-\left(\frac{3}{x^{2}}-\frac{1}{x}\right) \cos (x)-\frac{3}{x^{2}} \sin (x) .
$$

On the other hand, in the WH approximation

$$
\tan \eta_{2}(\varepsilon)=\frac{W_{d}}{2\left(\varepsilon-\varepsilon_{d}\right)} .
$$

From (14), it can be seen that the resonance scattering corresponds to $Y=1$, while the expression (19) gives the resonance at $\varepsilon=\varepsilon_{d}$. Thus, one gets

$$
\sqrt{\varepsilon_{d}} \int_{0}^{\infty} j_{2}\left(r \sqrt{\varepsilon_{d}}\right) n_{2}\left(r \sqrt{\varepsilon_{d}}\right) \omega(r) r^{2} \mathrm{~d} r=1 .
$$

For further calculations, we will use the exact relation

$$
\begin{gathered}
j_{2}(x) n_{2}(x)=\sin (x) \cos (x)\left[\frac{9}{x^{4}}-\left(\frac{3}{x^{3}}-\frac{1}{x}\right)\right] \\
+\left[\cos ^{2}(x)-\sin ^{2}(x)\right] \frac{3}{x^{2}}\left(\frac{3}{x^{3}}-\frac{1}{x}\right)
\end{gathered}
$$

and its approximation [12]:

$$
j_{2}(x) n_{2}(x)=-\frac{1}{5 x}, \quad x^{2}<2 .
$$

Inserting (3) into (20) and using (21), (22), one gets the two terms instead of the left part of $(20)$ :

$$
\begin{aligned}
Y_{1} & =-\frac{1}{5} \int_{0}^{R_{\mathrm{c}}} r \mathrm{~d} r\left[B_{1} \exp (-r / a)+B_{2} \exp (-0.5 r / a)\right] \\
& =\frac{z e^{2} a^{2}}{5 R_{\mathrm{c}}^{2}}\left[\left(R_{\mathrm{c}}-2 a\right) \exp \left(R_{\mathrm{c}} / a\right)+8\left(a-R_{\mathrm{c}}\right)\right. \\
& \left.\times \exp \left(0.5 R_{\mathrm{c}} / a\right)-6 a+5 R_{\mathrm{c}}+3 R_{\mathrm{c}}^{2} / a\right], \\
Y_{2} & =-\frac{z e^{2}}{\sqrt{\varepsilon_{d}}} \int_{\sqrt{\varepsilon_{d}} R_{\mathrm{c}}}^{\infty}\left[\cos \left(2 r_{\varepsilon}\right)\left(\frac{9}{r_{\varepsilon}^{4}}-\frac{3}{r_{\varepsilon}^{2}}\right)\right. \\
& \left.+\frac{1}{2} \sin \left(2 r_{\varepsilon}\right)\left(\frac{15}{r_{\varepsilon}^{3}}-\frac{9}{r_{\varepsilon}^{5}}-\frac{1}{r_{\varepsilon}}\right)\right] \mathrm{d} r_{\varepsilon} \\
& =-\frac{z e^{2}}{2 \sqrt{\varepsilon_{d}}}\left[\frac{3}{\varepsilon_{d} R_{\mathrm{c}}^{2}} \sin \left(2 \sqrt{\varepsilon_{d}} R_{\mathrm{c}}\right)\left(1-\frac{3}{8 \varepsilon_{d} R_{\mathrm{c}}^{2}}\right)\right. \\
& \left.+\frac{9}{2 \varepsilon_{d}^{3 / 2} R_{\mathrm{c}}^{3}} \cos \left(2 \sqrt{\varepsilon_{d}} R_{\mathrm{c}}\right)+\operatorname{Si}\left(2 \sqrt{\varepsilon_{d}} R_{\mathrm{c}}\right)-\frac{\pi}{2}\right], \quad(24)
\end{aligned}
$$

where $r_{\varepsilon}=r \sqrt{\varepsilon_{d}}$.

Then, (23), (24) and obvious condition

$$
Y_{1}+Y_{2}=1
$$

provide a connection between MP and $d$-electron parameters. Numerical solution of the system of Eqs. (23)-(25) allows to obtain the value of the parameter $a$ at fixed values of the rest parameters of the model.

In (23)-(25), we use the averaged over the hard-sphere (HS) reference system $\varepsilon_{d}$ which was obtained in [3]:

$$
\begin{aligned}
\left\langle\varepsilon_{d}\right\rangle_{\mathrm{HS}} & =\frac{225}{\pi^{2}}\left[\frac{2 \pi \rho r_{d}^{6}}{5 \sigma^{5}}\right. \\
& \left.+\frac{r_{d}^{6}}{\pi} \int_{0}^{\infty}\left[S_{\mathrm{HS}}(q)-1\right] q \mathrm{~d} q \int_{\sigma}^{\infty} \frac{\sin (q r)}{r^{7}} \mathrm{~d} r\right],
\end{aligned}
$$

where $\sigma$ is the HS diameter, $S_{\mathrm{HS}}(q)$ - the HS structure factor. 


\section{Results and discussion}

The procedure suggested in Sect. 4 may be applied to liquid Fe. The input magnitudes used for the calculation are listed in Table. The mean atomic volume, $\Omega$, is taken from the experiment [13], and the HS diameter - from the results of [3], $\nu=12$.

\section{TABLE}

Input data for the calculations.

\begin{tabular}{c|c|c|c|c|c|c}
\hline \hline & $z$ & $z_{d}$ & $R_{\text {c } \text { [a.u.] }}$ & $r_{d}$ [a.u.] & $\sigma$ [a.u.] & $\Omega$ [a.u.] \\
\hline $\mathrm{Fe}$ & 1.4 & 6.6 & 1.54 & 1.5 & 4.2 & 89.29
\end{tabular}

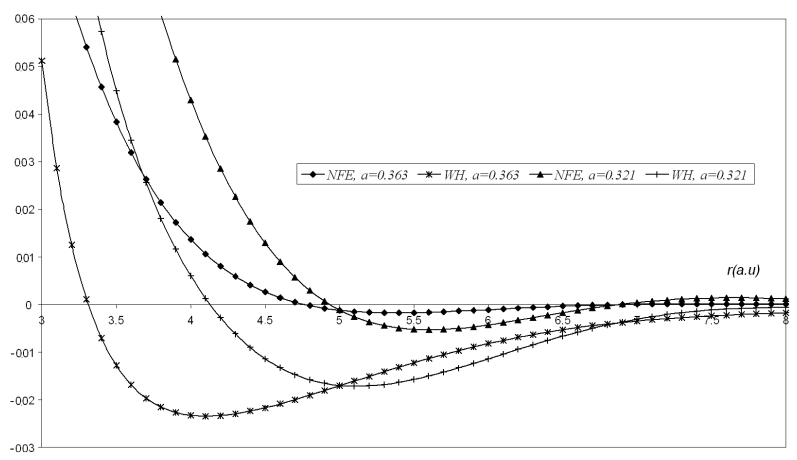

Fig. 1. $\varphi_{\mathrm{WH}}(r)$ and $\varphi_{\mathrm{NFE}}(r)$ (in a.u.) obtained by using the BS MP at different values of the parameter $a$.

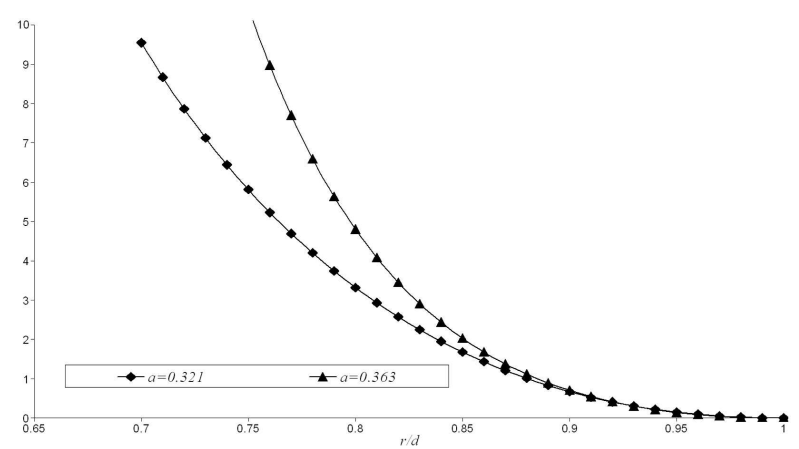

Fig. 2. Reduced repulsive part of the WH pair potential, $\beta\left[\varphi_{\mathrm{WH}}(r)-\varphi_{\mathrm{WH}}(d)\right]$, at different values of the parameter $a$.

In Fig. 1, the $\varphi_{\mathrm{WH}}(r)$ and $\varphi_{\mathrm{NFE}}(r)$ obtained by using the BS MP are presented for different values of the parameter $a$. The first value $a=0.363 \mathrm{a} . \mathrm{u}$. is taken from [5], and the second $a=0.321$ a.u. is calculated using (23)-(25).
From Fig. 1 it can be seen that the new value of the $a$ leads to the shift of the position of the first minimum of $\varphi_{\mathrm{WH}}(r)$ to the right. The same tendency for the liquid Fe was observed in [14] with the increase in the value of the parameter $r_{d}$. Such changing of the pair potential in [14] leads to the better agreement of the calculated internal and free energies with experiment data.

The softness of the pair interactions is estimated in Fig. 2, where the attractive parts of the pair potentials are presented in reduced coordinates $\beta[\varphi(r)-\varphi(d)]-$ $r / d$. Here, $d$ is the position of the first minimum of the pair potential, $\beta=1 / k_{b} T$ (temperature is taken equal to $1863 \mathrm{~K})$. One can see that the decrease in $a$ leads to the increase in the softness of the WH pair potential.

\section{Acknowledgments}

The author is grateful to Prof. L.D. Son for the fruitful collaboration and to the RFBR (grants 07-02-96045r and 08-03-00992) and the Russian Program of Scientific Schools (grant 4751.2008.3) for the financial support.

\section{References}

[1] J.M. Wills, W.A. Harrison, Phys. Rev. B 28, 4363 (1983).

[2] N.E. Dubinin, L.D. Son, N.A. Vatolin, Def. Diff. Forum 263, 105 (2007).

[3] N.E. Dubinin, L.D. Son, N.A. Vatolin, J. Phys., Condens. Matter 20, 114111 (2008).

[4] N.E. Dubinin, J. Phys., Conf. Series 144, 012115 (2009).

[5] J.L. Bretonnet, M. Silbert, Phys. Chem. Liq. 24, 169 (1992).

[6] G.M. Bhuiyan, I. Ali, S.M. Rahman, Physica B 334, 147 (2003).

[7] G.M. Bhuiyan, A.Z. Ziauddin Ahmed, Physica B 390, 377 (2007).

[8] Md. Saiful Alam, G.M. Bhuiyan, A.Z. Ziauddin Ahmed, R.I.M.A. Rashid, J. Phys., Conf. Series 98, 032006 (2008).

[9] B.A. Oli, Nuovo Cimento D 10, 891 (1988).

[10] J. Friedel, in: The Physics of Metals, Ed. J.M. Ziman, Cambridge University Press, New York 1969.

[11] W.A. Harrison, S. Froyen, Phys. Rev. B 21, 3214 (1980).

[12] L.I. Yastrebov, A.A. Katsnelson, The Base of the One-Electron Solid State Theory, Nauka, Moscow 1982 , p. 320.

[13] Y. Waseda, The Structure of Non-Crystalline Materials - Liquids and Amorphous Solids, McGraw-Hill, New York 1981.

[14] N.E. Dubinin, in: Proc. 5th Conf. "New Research Trends in Material Science", Sibiu (Romania) 200\%, Ed. W. Kappel, Bucharest 2007, p. 569. 